\title{
A quantum double construction in $\operatorname{Rel}^{\dagger}$
}

\author{
MASAHITO HASEGAWA \\ Research Institute for Mathematical Sciences, Kyoto University, Kyoto 606-8502, Japan \\ Email: hassei@kurims.kyoto-u.ac.jp \\ Last updated on 15 December 2010
}

We study bialgebras in the compact closed category Rel of sets and binary relations. Various monoidal categories with extra structure arise as the categories of (co)modules of bialgebras in Rel. In particular, for any group $G$ we derive a ribbon category of crossed $G$-sets as the category of modules of a Hopf algebra in Rel which is obtained by the quantum double construction. This category of crossed $G$-sets serves as a model of the braided variant of propositional linear logic.

\section{Introduction}

For last two decades it has been shown that there are plenty of important examples of traced monoidal categories (Joyal et al. 1996) and ribbon categories (tortile monoidal categories) (Shum 1994; Turaev 1994) in mathematics and theoretical computer science. In mathematics, most interesting ribbon categories are those of representations of quantum groups (quasi-triangular Hopf algebras) (Drinfel'd 1987; Kassel 1995) in the category of finite-dimensional vector spaces. In many of them, we have non-symmetric braidings (Joyal and Street 1993): in terms of the graphical presentation (Joyal and Street 1991; Selinger 2011), the braid $c=$ 久 is distinguished from its inverse $c^{-1}=\boldsymbol{X}$, and this is the key property for providing non-trivial invariants (or denotational semantics) of knots, tangles and so on (Freyd and Yetter 1989; Kassel 1995; Turaev 1994; Yetter 2001) as well as solutions of the quantum Yang-Baxter equation (Drinfel'd 1987; Kassel 1995), and 3dimensional topological quantum field theory (Bakalov and Kirilov 2001). In theoretical computer science, major examples include categories with fixed-point operators used in denotational and algebraic semantics (Bloom and Ésik 1993; Hasegawa 1999; Hasegawa 2009; Ştefănescu 2000), and the category of sets and binary relations and its variations used in models of linear logic (Girard 1987) and game semantics (Joyal 1977; Melliès 2004). Moreover, the Int-construction (Joyal et al. 1996) provides a rich class of models of Geometry of Interaction (Girard 1989; Abramsky et al. 2002; Haghverdi and Scott 2011) and more generally bi-directional information flow, including (Hildebrandt et al.

$\dagger$ This is a revised and expanded version of the work presented at the Conference on the Mathematical Foundations of Programming Semantics (MFPS XXVI) (Hasegawa 2010). 
2004; Katsumata 2008). In most of them, braidings are symmetric, hence $К$ is identified with $>$.

Although it is nice to know that all these examples share a common structure, it is also striking to observe that important examples from mathematics and those from computer science are almost disjoint ${ }^{\dagger}$. Is it just a matter of taste of mathematicians and computer scientists? Or is it the case that categories used in computer science cannot host structures interesting for mathematicians (non-symmetric braidings in particular)?

In this paper we demonstrate that we do have mathematically interesting structures in a category preferred by computer scientists. Specifically, we focus on the category Rel of sets and binary relations. Rel is a compact closed category (Kelly and Laplaza 1980), that is, a ribbon category in which braiding is symmetric and twist is trivial. We study bialgebras and Hopf algebras in Rel, and show that various monoidal categories with extra structure like traces and autonomy can be derived as the categories of (co)modules of bialgebras in Rel. As a most interesting example, for any group $G$ we consider the associated Hopf algebra in Rel, and apply the quantum double construction (Drinfel'd $1987)$ to it. The resulting Hopf algebra is equipped with a universal $R$-matrix as well as a universal twist. We show that the category of its modules is the category of crossed G-sets (Freyd and Yetter 1989; Whitehead 1949) and suitable binary relations, featuring non-symmetric braiding and non-trivial twist.

While the results mentioned above are interesting in their own right, we hope that this work serves as a useful introduction to the theory of quantum groups for researchers working on semantics of computation, and helps to connect these two research areas which deserve to interact much more.

\section{Related work}

Hopf algebras in connection to quantum groups (Drinfel'd 1987) have been extensively studied: standard references include (Kassel 1995; Majid 1995). The idea of using Hopf algebras for modelling various non-commutative linear logic goes back to Blute (Blute 1996), where the focus is on Hopf algebras in the *-autonomous category of topological vector spaces. As far as we know, there is no published result on Hopf algebras in Rel. Since Freyd and Yetter's work (Freyd and Yetter 1989), categories of crossed $G$-sets have appeared frequently as typical examples of braided monoidal categories. In the standard setting of finite-dimensional vector spaces, modules of the quantum double of a Hopf algebra $A$ amount to the crossed $A$-bimodules (Kassel 1995; Kassel and Turaev 1995), and our result is largely an adaptation of such a standard result to Rel. However we are not aware of a characterization of crossed $G$-sets in terms of a quantum double construction in the literature.

\footnotetext{
$\dagger$ An important exception would be dagger compact closed categories used in the study of quantum information protocols (Abramsky and Coecke 2004), though they do not feature non-symmetric braidings. We shall note that our category of crossed $G$-sets is actually a dagger tortile category in the sense of Selinger (Selinger 2011).
} 
Organization of this paper

In Section 2, we recall basic notions and facts on monoidal categories and bialgebras. In Section 3, we examine some bialgebras in Rel which arise from monoids and groups, and study the categories of (co)modules. Section 4 is devoted to a quantum double construction in Rel. In this development, we give a simplified description of the quantum double construction in terms of the Int-construction on traced symmetric monoidal categories. In Section 5 we observe that the ribbon Hopf algebra constructed in the previous section gives rise to a ribbon category of crossed $G$-sets, and look at some elements of this category. We discuss how this category can be used as a model of braided linear logic in Section 6. Section 7 concludes the paper.

\section{Monoidal categories and bialgebras}

\subsection{Monoidal categories}

A monoidal category (tensor category) (Mac Lane 1971; Joyal and Street 1993) $\mathcal{C}=$ $(\mathcal{C}, \otimes, I, a, l, r)$ consists of a category $\mathcal{C}$, a functor $\otimes: \mathcal{C} \times \mathcal{C} \rightarrow \mathcal{C}$, an object $I \in \mathcal{C}$ and natural isomorphisms $a_{A, B, C}:(A \otimes B) \otimes C \stackrel{\sim}{\rightarrow} A \otimes(B \otimes C), l_{A}: I \otimes A \stackrel{\sim}{\rightarrow} A$ and $r_{A}: A \otimes I \stackrel{\sim}{\rightarrow} A$ subject to the standard coherence diagrams. It is said to be strict if $a, l, r$ are the identity morphisms. For the sake of simplicity, in the most places in this paper we pretend that our monoidal categories are strict.

In the sequel, we will make use of the graphical presentation of morphisms in monoidal categories (Joyal and Street 1991; Selinger 2011). A morphism $f: A_{1} \otimes A_{2} \otimes \ldots \otimes A_{m} \rightarrow$ $B_{1} \otimes B_{2} \otimes \ldots \otimes B_{n}$ in a monoidal category will be drawn as (to be read from left to right):

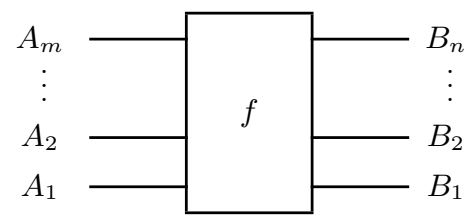

Morphisms can be composed, either sequentially or in parallel:
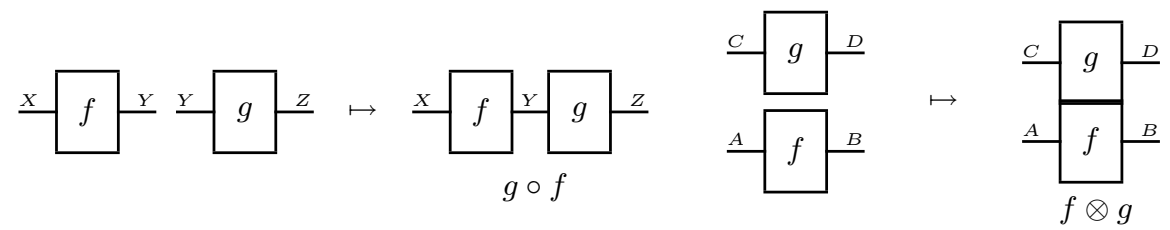

A braiding (Joyal and Street 1993) is a natural isomorphism $c_{A, B}: A \otimes B \stackrel{\sim}{\rightarrow} B \otimes A$ such that both $c$ and $c^{-1}$ satisfy the following "bilinearity" (the case for $c^{-1}$ is omitted):

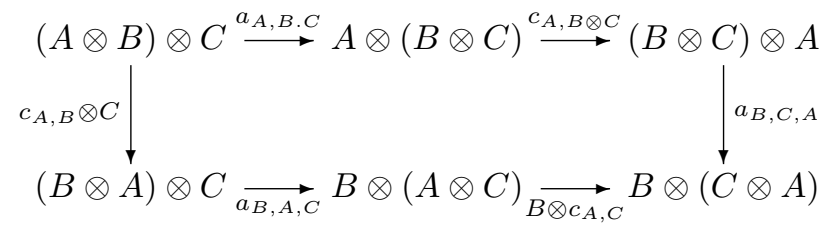


For braidings, we shall using the drawings $c_{A, B}={ }_{A} \nearrow^{B}{ }_{B}^{A}$ and $c_{A, B}^{-1}{ }^{A}{ }_{B}{ }^{B}{ }^{B}$.

A symmetry is a braiding such that $c_{A, B}=c_{B, A}^{-1}$. In that case we simply draw

hence $>>C$ A A braided/symmetric monoidal category is a monoidal category equipped with a braiding/symmetry.

A twist or a balance for a braided monoidal category is a natural isomorphism $\theta_{A}$ : $A \stackrel{\sim}{\rightarrow} A$ such that $\theta_{I}=i d_{I}$ and $\theta_{A \otimes B}=c_{B, A} \circ\left(\theta_{B} \otimes \theta_{A}\right) \circ c_{A, B}$ hold. Twists are drawn as $\theta_{A}=$ - - and $\theta_{A}^{-1}=\boldsymbol{c}$. A balanced monoidal category is a braided monoidal category with a twist. Note that a symmetric monoidal category is a balanced monoidal category with $\theta_{A}=i d_{A}$ for every $A$.

In a monoidal category, a dual pairing between two objects $A$ and $B$ is given by a pair of morphisms $d: I \rightarrow A \otimes B$, called unit, and $e: B \otimes A \rightarrow I$, called counit, drawn as $C_{A}^{B}$ and ${ }_{B} \longrightarrow$ respectively, satisfying

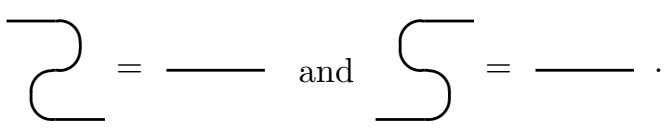

In such a dual pairing, $B$ is called the left dual of $A$, and $A$ is called the right dual of $B$. For an object, its left (or right) dual, if exists, is uniquely determined up to isomorphism. A monoidal category is left autonomous or left rigid if every object $A$ has a left dual $A^{*}$ with unit $\eta_{A}: I \rightarrow A \otimes A^{*}$ and counit $\varepsilon_{A}: A^{*} \otimes A \rightarrow I$. In a left autonomous category, $I^{*} \simeq I$ as well as $(A \otimes B)^{*} \simeq B^{*} \otimes A^{*}$ hold. Also $(-)^{*}$ extends to a contravariant functor, where, for a morphism $f: A \rightarrow B$, its dual $f^{*}: B^{*} \rightarrow A^{*}$ is given as:

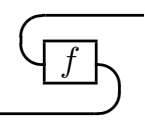

A ribbon category (Turaev 1994) (tortile monoidal category (Shum 1994)) is a balanced monoidal category which is left autonomous and moreover satisfies $\left(\theta_{A}\right)^{*}=\theta_{A^{*}}$. In a ribbon category, $(-)^{*}$ is a contravariant equivalence, and there is a natural isomorphism $A^{* *} \simeq A$ (hence the left dual of $A$ and the right dual of $A$ are isomorphic). Note that a ribbon category whose twist is the identity (and braiding is a symmetry) is a familiar compact closed category (Kelly and Laplaza 1980).

A traced monoidal category (Joyal et al. 1996) is a balanced monoidal category $\mathcal{C}$ equipped with a trace operator $\operatorname{Tr}_{A, B}^{X}: \mathcal{C}(A \otimes X, B \otimes X) \rightarrow \mathcal{C}(A, B)$ which will be drawn as a "feedback" operator

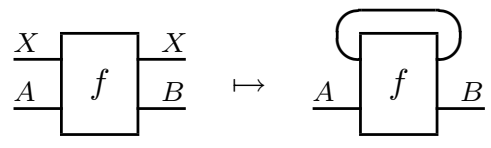

satisfying a few coherence axioms. Alternatively, by the structure theorem in ibid., traced monoidal categories are characterized as monoidal full subcategories of ribbon categories. Any ribbon category has a unique trace, called canonical trace (Joyal et al. 1996) (for uniqueness see e.g. (Hasegawa 2009)). For a morphism $f: A \otimes X \rightarrow B \otimes X$ in a ribbon 
category, its trace $\operatorname{Tr}_{A, B}^{X} f: A \rightarrow B$ is given by

$$
\operatorname{Tr}_{A, B}^{X} f=\left(i d_{B} \otimes\left(\varepsilon_{X} \circ\left(i d_{X^{*}} \otimes \theta_{X}\right) \circ c_{X, X^{*}}\right)\right) \circ\left(f \otimes i d_{X^{*}}\right) \circ\left(i d_{A} \otimes \eta_{X}\right) .
$$

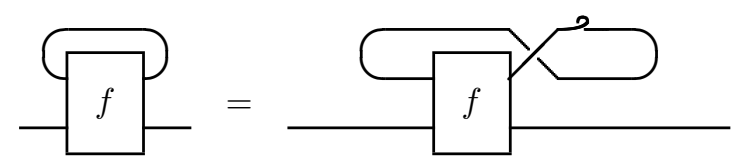

For monoidal categories $\mathcal{C}=(\mathcal{C}, \otimes, I, a, l, r)$ and $\mathcal{C}^{\prime}=\left(\mathcal{C}^{\prime}, \otimes^{\prime}, I^{\prime}, a^{\prime}, l^{\prime}, r^{\prime}\right)$, a monoidal functor from $\mathcal{C}$ to $\mathcal{C}^{\prime}$ is a tuple $\left(F, m, m_{I}\right)$ where $F$ is a functor from $\mathcal{C}$ to $\mathcal{C}^{\prime}, m$ is a natural transformation from $F(-) \otimes^{\prime} F(=)$ to $F(-\otimes=)$ and $m_{I}: I^{\prime} \rightarrow F I$ is an arrow in $\mathcal{C}^{\prime}$, satisfying three coherence conditions. It is called strong if $m_{A, B}$ and $m_{I}$ are all isomorphisms, and strict if they are all identities. A balanced monoidal functor from a balanced $\mathcal{C}$ to another $\mathcal{C}^{\prime}$ is a monoidal functor $\left(F, m, m_{I}\right)$ which additionally satisfies $m_{B, A} \circ c_{F A, F B}=F c_{A, B} \circ m_{A . B}$ and $F\left(\theta_{A}\right)=\theta_{F A}$.

For monoidal functors $\left(F, m, m_{I}\right),\left(G, n, n_{I}\right)$ with the same source and target monoidal categories, a monoidal natural transformation from $\left(F, m, m_{I}\right)$ to $\left(G, n, n_{I}\right)$ is a natural transformation $\varphi: F \rightarrow G$ such that $\varphi_{A \otimes B} \circ m_{A, B}=n_{A, B} \circ \varphi_{A} \otimes \varphi_{B}$ and $\varphi_{I} \circ m_{I}=n_{I}$ hold. A (balanced/symmetric) monoidal adjunction between (balanced/symmetric) monoidal categories is an adjunction in which both of the functors are (balanced/symmetric) monoidal and the unit and counit are monoidal natural transformations.

\subsection{Monoids, comonoids and (co)modules}

A monoid in a monoidal category $\mathcal{C}=(\mathcal{C}, \otimes, I, a, l, r)$ is an object $A$ equipped with morphisms $m: A \otimes A \rightarrow A$, called the multiplication, and $1: I \rightarrow A$, called the unit, such that the following diagrams commute.
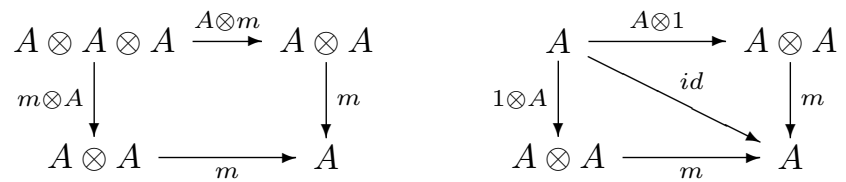

With notations $m=\square-$ and $1=\bigcirc-$, these diagrams can be expressed as follows.

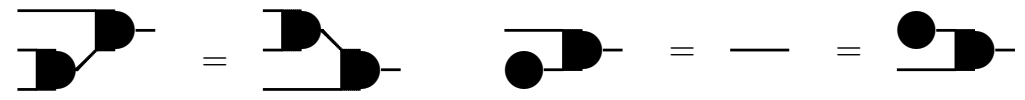

When $\mathcal{C}$ is symmetric and $m \circ c_{A, A}=m$, i.e., $\square-=\square-$ holds, we say $A$ is commutative.

Dually, a comonoid in a monoidal category $\mathcal{C}$ is an object $A$ equipped with morphisms $\Delta: A \rightarrow A \otimes A$, called the comultiplication, and $\epsilon: A \rightarrow I$, called the counit, satisfying
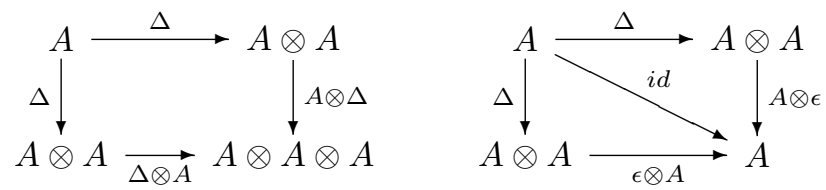

They can be drawn as 

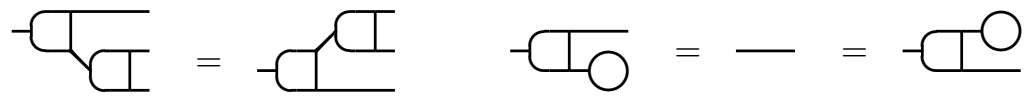

where $\Delta=\neg$ and $\epsilon=-\bigcirc$. When $\mathcal{C}$ is symmetric and $c_{A, A} \circ \Delta=\Delta$ holds (graphically: $\square=-\square \times$ ), we say $A$ is co-commutative.

Suppose that $A=(A, m, 1)$ is a monoid. $A$ gives rise to a monad $A \otimes(-)$ whose multiplication is $m \otimes X: A \otimes A \otimes X \rightarrow A \otimes X$ and unit is $1 \otimes X: X \rightarrow A \otimes X$. An $A$-module is an Eilenberg-Moore algebra of this monad. More explicitly, an $A$-module consists of an object $X$ and a morphism $\alpha: A \otimes X \rightarrow X$, called the action, satisfying
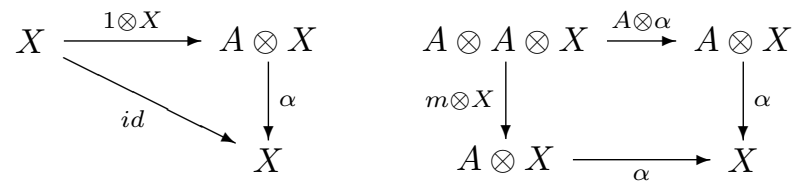

or, in the graphical presentation,
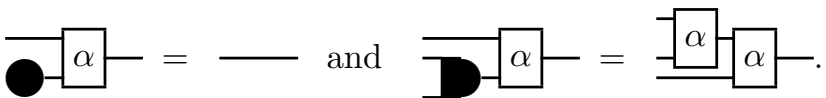

A morphism of $A$-modules from $(X, \alpha)$ to $(Y, \beta)$ is a morphism $f: X \rightarrow Y$ satisfying

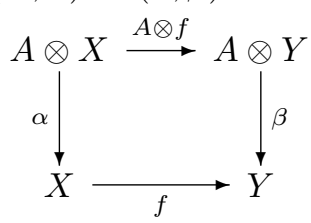

Let us denote the category of $A$-modules and morphisms by $\operatorname{Mod}(A)$.

Dually, given a comonoid $A=(A, \Delta, \epsilon)$, an $A$-comodule is an Eilenberg-Moore coalgebra of the comonad $A \otimes(-)$ whose comultiplication is $\Delta \otimes X: A \otimes X \rightarrow A \otimes A \otimes X$ and counit is $\epsilon \otimes X: A \otimes X \rightarrow X$. Explicitly, an $A$-comodule consists of an object $X$ and a morphism $\alpha: X \rightarrow A \otimes X$, called the coaction, satisfying the axioms dual to those of modules. A morphism of $A$-comodules from $(X, \alpha)$ to $(Y, \beta)$ is then a morphism $f: X \rightarrow Y$ making the evident diagram commute. We will denote the category of $A$-comodules and morphisms by $\operatorname{Comod}(A)$.

\subsection{Bialgebras and Hopf algebras}

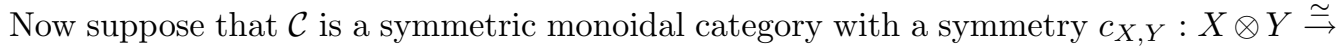
$Y \otimes X$. A bialgebra in $\mathcal{C}$ is given by a tuple $A=(A, m, 1, \Delta, \epsilon)$ where $A$ is an object of $\mathcal{C}$ and $(A, m, 1)$ is a monoid in $\mathcal{C}$ while $(A, \Delta, \epsilon)$ is a comonoid in $\mathcal{C}$, satisfying
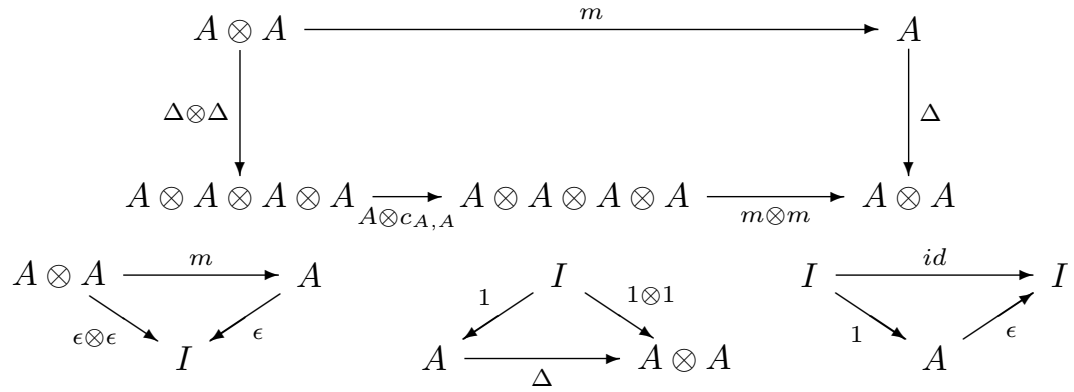

Graphically: 


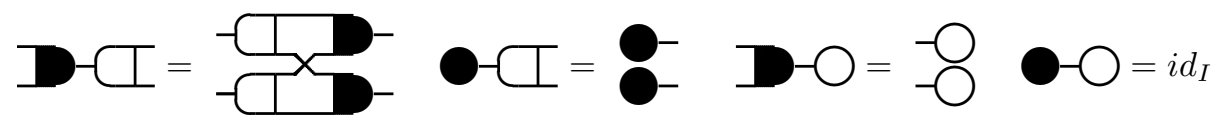

We say $A$ is commutative (resp. co-commutative) when it is commutative (resp. cocommutative) as a monoid (resp. comonoid). For a bialgebra $A$, we can consider the category of modules $\operatorname{Mod}(A)$ as well as that of comodules $\operatorname{Comod}(A)$. The functor $A \otimes$ $(-)$ is both monoidal and comonoidal. Moreover, as a monad $A \otimes(-)$ is comonoidal, while as a comonad it is monoidal. It follows that (cf. (Bruguieres and Virelizier 2006; Pastro and Street 2009)) both $\operatorname{Mod}(A)$ and $\operatorname{Comod}(A)$ are monoidal categories. Explicitly, in $\operatorname{Mod}(A)$, the tensor unit is $(I, A \otimes I \simeq A \stackrel{\epsilon}{\rightarrow} I)$ and the tensor product of $(X, \alpha)$ and $(Y, \beta)$ is

$$
\left(X \otimes Y, A \otimes X \otimes Y \stackrel{\Delta \otimes X \otimes Y}{\longrightarrow} A \otimes A \otimes X \otimes Y \stackrel{A \otimes c_{A, X} \otimes Y}{\longrightarrow} A \otimes X \otimes A \otimes Y \stackrel{\alpha \otimes \beta}{\longrightarrow} X \otimes Y\right) .
$$

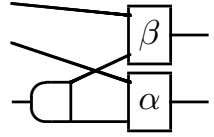

The monoidal structure of $\operatorname{Comod}(A)$ is given by dualizing that of $\operatorname{Mod}(A)$.

A Hopf algebra is a bialgebra $A=(A, m, 1, \Delta, \epsilon)$ equipped with a morphism $S: A \rightarrow A$, called an antipode, such that

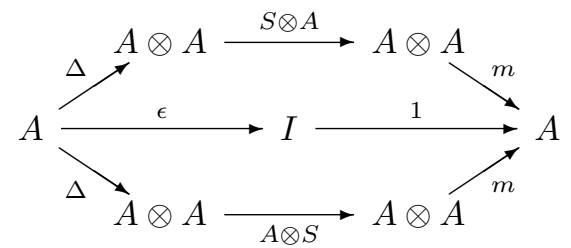

commutes (see the picture below).

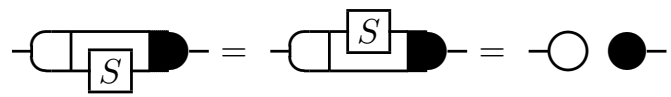

The antipode of a Hopf algebra is unique. In general, an antipode does not have to be invertible (that is, an isomorphism); see (Takeuchi 1971) for some examples. It is wellknown that the antipode $S$ of any commutative or co-commutative Hopf algebra satisfies $S \circ S=i d$, hence is invertible. It is also known that any Hopf algebra in a compact closed category with equalizers has an invertible antipode (Takeuchi 1999), and it is the case for the category of finite dimensional vector spaces. All concrete examples considered below have an invertible antipode (see also Remark 3.2).

Lemma 2.1. If $\mathcal{C}$ is a compact closed category and $A$ is a Hopf algebra in $\mathcal{C}$, then $\operatorname{Mod}(A)$ is left autonomous, where a left dual of a module $(X, \alpha)$ is

$$
A \otimes X^{*} \stackrel{c}{\rightarrow} X^{*} \otimes A \stackrel{X^{*} \otimes S \otimes \eta}{\longrightarrow} X^{*} \otimes A \otimes X \otimes X^{*} X^{*} \stackrel{\otimes \alpha \otimes X^{*}}{\longrightarrow} X^{*} \otimes X \otimes X^{*} \stackrel{\varepsilon \otimes X^{*}}{\longrightarrow} X^{*} .
$$

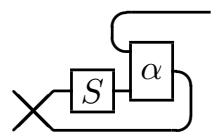


The unit and counit of the dual pairing are given by the unit and counit of the dual pairing of $X$ and $X^{*}$ in $\mathcal{C}$.

Remark 2.1. In this paper we only consider bialgebras and Hopf algebras in symmetric monoidal categories. However, it completely makes sense to think about bialgebras and Hopf algebras in braided monoidal categories, and it is the central topic in (Majid 1994).

Remark 2.2. As noted in (Cockett and Seely 1997), the category of modules of a bialgebra in a symmetric or braided linearly distributive category is a linearly distributive category. Similarly, the category of modules of a Hopf algebra in a symmetric or braided *-autonomous category is a *-autonomous category.

\subsection{Braiding and twist on (co)modules of a bialgebra}

If a bialgebra $A$ is co-commutative (resp. commutative), the monoidal category $\operatorname{Mod}(A)$ $(\operatorname{resp} . \operatorname{Comod}(A))$ has a symmetry inherited from the base symmetric monoidal category. However, (whether $A$ is (co-)commutative or not) there can be some non-trivial braiding and twist on $\operatorname{Mod}(A)$ or $\operatorname{Comod}(A)$ : we shall look at the case of $\operatorname{Mod}(A)$. Suppose that $\operatorname{Mod}(A)$ is braided with a braiding $\sigma$ (while we use $c$ for the symmetry of the base symmetric monoidal category). Since $A=(A, m)$ is an $A$-module, we have $\sigma_{A, A}$ : $A \otimes A \rightarrow A \otimes A$, and $c_{A, A} \circ \sigma_{A, A} \circ(1 \otimes 1): I \rightarrow A \otimes A$ which we shall denote by $R$. Conversely, from this $R: I \rightarrow A \otimes A$ we can recover $\sigma_{X, Y}: X \otimes Y \rightarrow Y \otimes X$ for modules $X=(X, \alpha)$ and $Y=(Y, \beta)$ as

$$
X \otimes Y \stackrel{R \otimes X \otimes Y}{\longrightarrow} A \otimes A \otimes X \otimes Y \stackrel{A \otimes c_{A, X} \otimes Y}{\longrightarrow} A \otimes X \otimes A \otimes Y \stackrel{\alpha \otimes \beta}{\longrightarrow} X \otimes Y \stackrel{c_{X, Y}}{\longrightarrow} Y \otimes X
$$

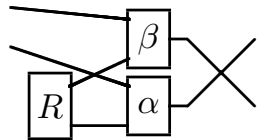

provided the base symmetric monoidal category $\mathcal{C}$ is closed and the global section functor $\mathcal{C}(I,-): \mathcal{C} \rightarrow$ Set is faithful - this is the case for all commonly used examples, including the category of vector spaces and linear maps, as well as Rel. In such cases there is a bijective correspondence between braidings on $\operatorname{Mod}(A)$ and morphisms of $I \rightarrow A \otimes A$ satisfying certain equations (Kassel 1995; Majid 1995; Street 2007). Such a morphism of $I \rightarrow A \otimes A$ is called a universal $R$-matrix or a braiding element. Explicitly, a universal $R$-matrix is a morphism $R: I \rightarrow A \otimes A$ which is convolution-invertible (there exists $R^{\circ}: I \rightarrow A \otimes A$ satisfying $(m \otimes m) \circ\left(A \otimes c_{A, A} \otimes A\right) \circ\left(R \otimes R^{\circ}\right)=(m \otimes m) \circ\left(A \otimes c_{A, A} \otimes\right.$ $\left.A) \circ\left(R^{\circ} \otimes R\right)=1 \otimes 1\right)$ and satisfies the following three equations.

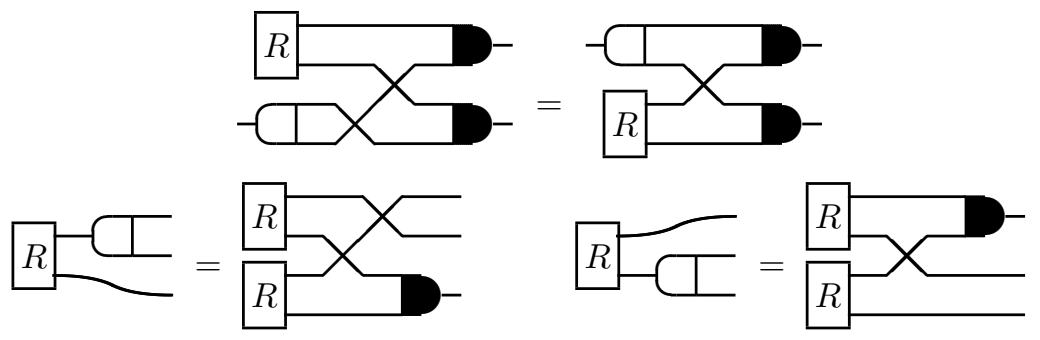


The convolution-invertibility ensures the invertibility of the braid $\sigma$ induced from $R$. These three graphically presented equations imply that $\sigma$ is a morphism of modules, that $\sigma$ is bilinear, and that $\sigma^{-1}$ is bilinear, respectively. A bialgebra equipped with a universal $R$-matrix is called a quasi-triangular bialgebra.

Next, let $A$ be a quasi-triangular Hopf algebra in a compact closed category and suppose that $\operatorname{Mod}(A)$ is a ribbon category, i.e., not just braided but also with a twist $\theta$. We then have a morphism $v=\theta_{A} \circ 1: I \rightarrow A$, from which we can recover $\theta_{X}$ : $X \rightarrow X$ for a module $X=(X, \alpha)$ as $X \stackrel{v \otimes X}{\longrightarrow} A \otimes X \stackrel{\alpha}{\longrightarrow} X$ provided the global section functor of the base compact closed category is faithful. In such cases we have a bijective correspondence between twists on $\operatorname{Mod}(A)$ and certain morphisms $v: I \rightarrow A$ satisfying a few axioms (Kassel 1995; Majid 1995; Turaev 1994). Such a $v$ is called a universal twist or a twist element. Explicitly, a universal twist is a morphism $v: I \rightarrow A$ which is convolution-invertible (there exists $v^{\circ}: I \rightarrow A$ such that $m \circ\left(v \otimes v^{\circ}\right)=1$ ), central $(m \circ(A \otimes v)=m \circ(v \otimes A))$ and satisfies the following three equations.

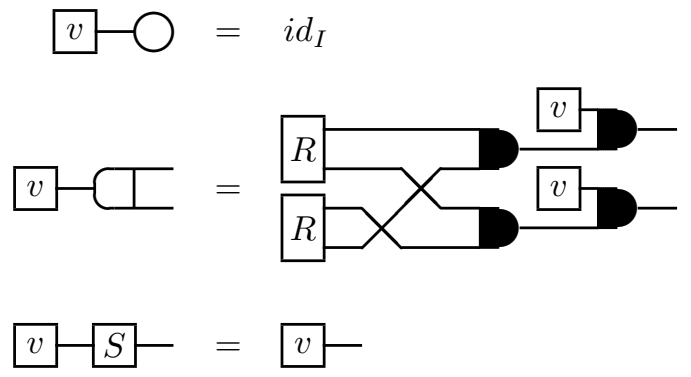

The convolution-invertibility implies that $\theta$ induced from $v$ is invertible, and centrality says that $\theta$ is a morphism of modules. The first two graphical equations amount to two axioms for twists, while the last one is required for the axiom $\left(\theta_{X}\right)^{*}=\theta_{X^{*}}$. A quasitriangular Hopf algebra equipped with a universal twist is called a ribbon Hopf algebra. In summary, we have the following results:

Proposition 2.1. (Turaev 1994; Kassel 1995; Yetter 2001)

1 If $A$ is a quasi-triangular bialgebra in a symmetric monoidal category $\mathcal{C}$, then $\operatorname{Mod}(A)$ is a braided monoidal category.

2 If $A$ is a ribbon Hopf algebra in a compact closed category $\mathcal{C}$, then $\operatorname{Mod}(A)$ is a ribbon category.

We will give a non-commutative non-co-commutative ribbon Hopf algebra in Rel in Section 4.

\subsection{Examples}

We shall look at a few basic cases.

Example 2.1. As a classical example, let us consider the category Vect $_{k}$ of vector spaces over a field $k$ and linear maps. Vect $_{k}$ is a symmetric monoidal category whose monoidal product is given by the tensor product of vector spaces, and $k$ (the 
1-dimensional space) serves as the tensor unit. Its full subcategory Vect ${ }_{k}^{\text {fin }}$ of finite dimensional vector spaces is a compact closed category; for a finite dimensional $V$, its left (and right) dual is the dual vector space $V^{*}=\operatorname{hom}(V, k)$ of linear maps from $V$ to $k$, with unit given by the dual basis and counit the evaluation map. A monoid in Vect ${ }_{k}$ is nothing but an algebra in the standard sense. Similarly, a comonoid in Vect $_{k}$ is what is normally called a coalgebra. Modules, comodules, bialgebras and Hopf algebras in Vect ${ }_{k}$ and Vect $_{k}^{\text {fin }}$ are exactly those in the classical sense; a detailed account can be found in (Kassel 1995).

Example 2.2. Let Set be the category of sets and functions. By taking finite products as tensor products, Set forms a symmetric monoidal category. A monoid in Set is just a monoid in the usual sense. For any set $X$, the diagonal map $X \rightarrow X \times X$ and the terminal map $X \rightarrow 1$ give a commutative comonoid structure on $X-$ and this is the unique comonoid structure on $X$. Given a monoid $M$, its modules are just the $M$-sets, i.e., sets on which $M$ acts, and $\operatorname{Mod}(M)$ is isomorphic to the category $M$-Set of $M$-sets and functions respecting $M$-actions. For any set $X$, a comodule $(A, \alpha: A \rightarrow X \times A)$ of the unique comonoid $X=(X, \Delta, \epsilon)$ on $X$ is determined by the function $\pi \circ \alpha: A \rightarrow X$, and $\operatorname{Comod}(X)$ is isomorphic to the slice category Set $/ X$. A bialgebra in Set is a monoid equipped with the unique comonoid structure. A Hopf algebra in Set is then a group with the unique comonoid structure, where the antipode is given by the inverse $(-)^{-1}$.

\section{Bialgebras in Rel}

Now let us turn our attention to the category Rel of sets and binary relations. Rel is a compact closed (hence ribbon) category, where the tensor product of sets $X$ and $Y$ is given by the direct product $X \times Y$ of sets and the unit object is a singleton set $I=\{*\}$. For a set $X$, its left dual $X^{*}$ is $X$ itself, with unit and counit given by

$$
\begin{aligned}
& \eta_{X}=\{(*,(x, x)) \mid x \in X\}: I \rightarrow X \times X, \\
& \varepsilon_{X}=\{((x, x), *) \mid x \in X\}: X \times X \rightarrow I .
\end{aligned}
$$

\subsection{Bialgebras and Hopf algebras inherited from Set}

The easiest cases of bialgebras and Hopf algebras in Rel are those arising from monoids and groups in Set, respectively. First, we shall note that there is an identity-on-object, strict symmetric monoidal functor $J$ : Set $\rightarrow$ Rel sending a set to itself and a function $f: X \rightarrow Y$ to a binary relation $\{(x, f(x)) \mid x \in X\}$ from $X$ to $Y$, and recall a standard result:

Lemma 3.1. A strong symmetric monoidal functor preserves the structure of monoids, comonoids, bialgebras and Hopf algebras. 
From this and Example 2.2, it follows that a monoid $M=(M, \cdot, e)$ (in Set) gives rise to a co-commutative bialgebra $\bar{M}=(M, m, 1, \Delta, \epsilon)$ in Rel, with

$$
\begin{aligned}
m & =\left\{\left(\left(a_{1}, a_{2}\right), a_{1} \cdot a_{2}\right) \mid a_{1}, a_{2} \in M\right\}: M \times M \rightarrow M \\
1 & =\{(*, e)\}: I \rightarrow M \\
\Delta & =\{(a,(a, a)) \mid a \in M\}: M \rightarrow M \times M \\
\epsilon & =\{(a, *) \mid a \in M\}: M \rightarrow I .
\end{aligned}
$$

$\bar{M}$ is commutative if $M$ is commutative. Similarly, a group $G=\left(G, \cdot, e,(-)^{-1}\right)$ gives rise to a co-commutative Hopf algebra $\bar{G}=(G, m, 1, \Delta, \epsilon, S)$ in Rel, with an antipode $S=\left\{\left(g, g^{-1}\right) \mid g \in G\right\}: G \rightarrow G$.

Let us examine the category $\operatorname{Mod}(\bar{G})$ for a group $G=\left(G, \cdot, e,(-)^{-1}\right)$ (it makes sense to think about $\operatorname{Mod}(\bar{M})$ for a monoid $M$, but when $M$ is not a group the description of $\operatorname{Mod}(\bar{M})$ can be rather complicated). A module of $\bar{G}$ is a set $X$ equipped with a binary relation $\alpha: G \times X \rightarrow X$ subject to the two axioms given before. It is not hard to see that $\alpha$ is actually a function, in fact a $G$-action on $X$ : for $g \in G$ and $x \in X$, by letting $g \bullet x$ be the unique $x^{\prime} \in X$ such that $\left((g, x), x^{\prime}\right) \in \alpha$, we have $e \bullet x=x$ and $(g \cdot h) \bullet x=g \bullet(h \bullet x)$. Therefore we can identify objects of $\operatorname{Mod}(\bar{G})$ with $G$-sets: a morphism from a $G$-set $(X, \bullet)$ to $(Y, \bullet)$ is then a binary relation $r: X \rightarrow Y$ such that $(x, y) \in r$ implies $(g \bullet x, g \bullet y) \in r$. Since $\bar{G}$ is a co-commutative Hopf algebra, $\operatorname{Mod}(\bar{G})$ is a compact closed category which is actually very similar to Rel. Explicitly, the tensor of $(X, \bullet)$ and $(Y, \bullet)$ is $(X \times Y,(g,(x, y)) \mapsto(g \bullet x, g \bullet y))$, while the tensor unit is $(\{*\},(g, x) \mapsto *)$. A left dual of $(X, \bullet)$ is $(X, \bullet)$ itself.

Next, we shall look at $\operatorname{Comod}(\bar{M})$ for a monoid $M=(M, \cdot, e)$. A comodule of $\bar{M}$ is a set $X$ with a binary relation $\alpha: X \rightarrow M \times X$ subject to the comodule axioms - but the axioms imply that $\alpha$ is a function whose second component is the identity on $X$. Hence an object of $\operatorname{Comod}(\bar{M})$ can be identified with a set $X$ equipped with a function $|-|: X \rightarrow M$; a morphism from $\left(X,||_{-}\right)$to $\left(Y,||_{-}\right)$is then a binary relation $r: X \rightarrow Y$ such that $(x, y) \in r$ implies $|x|=|y|$. $\operatorname{Comod}(\bar{M})$ is a monoidal category, with $(X,|-|) \otimes(Y,|-|)=(X \times Y,(x, y) \mapsto|x| \cdot|y|)$ and $I=(\{*\}, x \mapsto e)$.

\section{Proposition 3.1.}

1 If $G$ is a group, every object $(X,|-|)$ of $\operatorname{Comod}(\bar{G})$ has a left dual $\left(X,\left.\left.\right|_{-}\right|^{-1}\right)$ (and $\operatorname{Comod}(\bar{G})$ is pivotal (Freyd and Yetter 1989)).

2 If $G$ is an Abelian group, $\operatorname{Comod}(\bar{G})$ is a compact closed category.

3 If $M$ is a commutative monoid, $\operatorname{Comod}(\bar{M})$ is symmetric monoidal.

4 If $M$ is a commutative cancellable monoid, $\operatorname{Comod}(\bar{M})$ is a traced symmetric monoidal category.

5 If $M$ is a left (resp. right)-cancellable monoid, $\operatorname{Comod}(\bar{M})$ has a left (resp. right) trace in the sense of Selinger (Selinger 2011).

Thus we can derive a number of monoidal categories with symmetry, duals, and trace as categories of (co)modules of (the associated bialgebra of) a monoid or a group. However, they do not have a non-symmetric braiding; in Section 4 we give a Hopf algebra in Rel whose category of modules has a non-symmetric braiding and a non-trivial twist. 


\subsection{Some constructions}

There are a number of ways of constructing bialgebras and Hopf algebras in Rel from the existing ones. Here we shall look at some basic constructions.

Opposite bialgebras and Hopf algebras Given a bialgebra $A=(A, m, 1, \Delta, \epsilon)$ in Rel, its opposite bialgebra is the bialgebra $A^{\mathrm{op}}=\left(A, m^{\mathrm{op}}, 1, \Delta, \epsilon\right)$ where

$$
m^{\mathrm{op}}=m \circ c_{A, A}=\left\{\left(\left(x_{2}, x_{1}\right), y\right) \mid\left(\left(x_{1}, x_{2}\right), y\right) \in m\right\} .
$$

If $A=(A, m, 1, \Delta, \epsilon, S)$ is a Hopf algebra with an invertible antipode $S$, its opposite Hopf-algebra is the Hopf algebra $A^{\mathrm{op}}=\left(A, m^{\mathrm{op}}, 1, \Delta, \epsilon, S^{-1}\right)$.

Dual bialgebras and Hopf algebras Given a bialgebra $A=(A, m, 1, \Delta, \epsilon)$ in Rel, its dual bialgebra is the bialgebra $A^{*}=\left(A, \Delta^{*}, \epsilon^{*}, m^{*}, 1^{*}\right)$ where

$$
\begin{aligned}
\Delta^{*} & =\left\{\left(\left(y_{2}, y_{1}\right), x\right) \mid\left(x,\left(y_{1}, y_{2}\right)\right) \in \Delta\right\} \\
\epsilon^{*} & =\{(*, x) \mid(x, *) \in \epsilon\} \\
m^{*} & =\left\{\left(y,\left(x_{2}, x_{1}\right)\right) \mid\left(\left(x_{1}, x_{2}\right), y\right) \in m\right\} \\
1^{*} & =\{(y, *) \mid(*, y) \in 1\} .
\end{aligned}
$$

If $A$ is a Hopf algebra with antipode $S$, then $A^{*}$ is a Hopf algebra with antipode $S^{*}=$ $\{(y, x) \mid(x, y) \in S\}$.

Tensor products When $A_{1}=\left(A_{1}, m_{1}, 1_{1}, \Delta_{1}, \epsilon_{1}\right)$ and $A_{2}=\left(A_{2}, m_{2}, 1_{2}, \Delta_{2}, \epsilon_{2}\right)$ are bialgebras in Rel, their tensor product is the bialgebra $A_{1} \otimes A_{2}=\left(A_{1} \times A_{2}, m_{12}, 1_{12}, \Delta_{12}, \epsilon_{12}\right)$ where

$$
\begin{aligned}
& m_{12}=\left\{\left(\left(\left(x_{1}, x_{2}\right),\left(y_{1}, y_{2}\right)\right),\left(z_{1}, z_{2}\right)\right) \mid\left(\left(x_{i}, y_{i}\right), z_{i}\right) \in m_{i}\right\} \\
& 1_{12}=\left\{\left(*,\left(x_{1}, x_{2}\right)\right) \mid x_{i} \in 1_{i}\right\} \\
& \Delta_{12}=\left\{\left(\left(x_{1}, x_{2}\right),\left(\left(y_{1}, y_{2}\right),\left(z_{1}, z_{2}\right)\right)\right) \mid\left(x_{i},\left(y_{i}, z_{i}\right)\right) \in \Delta_{i}\right\} \\
& \epsilon_{12}=\left\{\left(\left(x_{1}, x_{2}\right), *\right) \mid\left(x_{i}, *\right) \in \epsilon_{i}\right\} .
\end{aligned}
$$

When both $A_{1}$ and $A_{2}$ are Hopf algebras with antipode $S_{1}$ and $S_{2}$ respectively, then $A_{1} \otimes A_{2}$ is a Hopf algebra with antipode $S_{12}=\left\{\left(\left(x_{1}, x_{2}\right),\left(y_{1}, y_{2}\right)\right) \mid\left(x_{i}, y_{i}\right) \in S_{i}\right\}$.

By these constructions, one can construct a non-commutative non-co-commutative bialgebras and Hopf algebras. For example, for a non-Abelian group $G, \bar{G} \otimes \bar{G}^{*}$ is a Hopf algebra which is neither commutative nor co-commutative. However, this Hopf algebra does not have an $R$-matrix - for which we need a more sophisticated construction, and it is the topic of the next section.

Remark 3.1. Of course, there are lots of bialgebras and Hopf algebras in Rel which cannot be obtained by these constructions on $\bar{M}$ 's or $\bar{G}$ 's. For an easy example, let $X$ be a set and $(M X, \oplus, 0)$ be the free commutative monoid on $X$; or, equivalently, let $M X$ be the set of finite multisets of elements of $X, \oplus$ the union of multisets, and 0 the empty multiset. Then there is a bialgebra $M X=(M X, m, 1, \Delta, \epsilon)$ in Rel where $m=\left\{\left(\left(x_{1}, x_{2}\right), x_{1} \oplus x_{2}\right) \mid x_{i} \in M X\right\}, 1=\{(*, 0)\}, \Delta=\left\{\left(x_{1} \oplus x_{2},\left(x_{1}, x_{2}\right)\right) \mid x_{i} \in M X\right\}$ and $\epsilon=\{(0, *)\}$. Obviously $M X^{\text {op }}$ and $M X^{*}$ are isomorphic to $M X$. 
Remark 3.2. As of writing this paper, we do not know if all Hopf algebras in Rel have an invertible antipode. Note that Rel does not have all equalizers, so the result in (Takeuchi 1999) cannot be applied to Rel. On the other hand, it is not clear if the construction of a Hopf algebra with a non-invertible antipode in (Takeuchi 1999) can be carried out in Rel.

\section{A quantum double construction in Rel}

In the previous section, we have observed that every group $G=\left(G, \cdot, e,(-)^{-1}\right)$ gives rise to a co-commutative Hopf algebra $\bar{G}=(G, m, 1, \Delta, \epsilon, S)$ in Rel. For obtaining a quasi-triangular Hopf algebra, in this section we shall apply Drinfel'd's quantum double construction (Drinfel'd 1987; Majid 1990) to $\bar{G}$.

\subsection{Quantum double construction in compact and traced categories}

We shall use the quantum double construction given in terms of Hopf algebras in compact closed categories:

Proposition 4.1. (cf. (Chen 2000; Kassel 1995; Kassel and Turaev 1995)) Suppose that $\mathcal{C}$ is a compact closed category and $A=(A, m, 1, \Delta, \epsilon, S)$ is a Hopf algebra in $\mathcal{C}$, where the antipode $S$ is invertible. Then there exists a quasi-triangular Hopf algebra $D(A)$ on $A^{*} \otimes A$.

Before going into the technical detail, let us first explain an outline of the construction and give some informal remarks. Given a Hopf algebra $A=(A, m, 1, \Delta, \epsilon, S)$ with $S$ invertible, let $A^{\mathrm{op} *}=\left(A^{*}, \Delta^{*}, \epsilon^{*},\left(m^{\mathrm{op}}\right)^{*}, 1^{*},\left(S^{-1}\right)^{*}\right)$ be the dual opposite Hopf algebra. It follows that there are suitable actions of $A$ on $A^{\text {op* }}$ and $A^{\text {op* } *}$ on $A$, and with them we can form a bicrossed product (Majid 1990; Majid 1995) of $A^{\mathrm{op} *}$ with $A$, which is the Hopf algebra $D(A)$. We shall note that $D(A)$ is almost like a tensor product of $A^{\text {op* }}$ with $A$ itself - except some clever adjustment on the multiplication and antipode. Also let us remark that $\operatorname{Mod}\left(A^{\mathrm{op} *}\right)$ is isomorphic to $\operatorname{Comod}(A)$, and $\operatorname{Mod}(D(A))$ can be regarded as a combination of $\operatorname{Comod}(A)$ and $\operatorname{Mod}(A)$, as we will soon see for the case of $\bar{G}$ in Rel below.

Unfortunately, a direct description of $D(A)$ is rather complicated; see (Chen 2000) for instance. Instead, we shall give an alternative, simpler description using the Intconstruction of Joyal, Street and Verity (Joyal et al. 1996).

Recall that, for a traced monoidal category $\mathcal{C}$, one can construct a ribbon category $\operatorname{Int}(\mathcal{C})$ whose objects are pairs of those of $\mathcal{C}$, and a morphism $f:\left(A_{+}, A_{-}\right) \rightarrow\left(B_{+}, B_{-}\right)$ in $\operatorname{Int}(\mathcal{C})$ is a morphism from $A_{+} \otimes B_{-}$to $B_{+} \otimes A_{-}$in $\mathcal{C}$ which can be drawn as

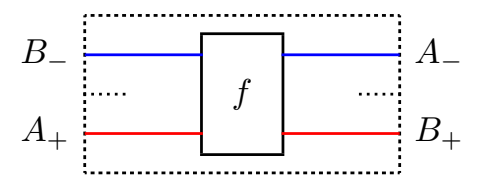

The composition of $f:\left(A_{+}, A_{-}\right) \rightarrow\left(B_{+}, B_{-}\right)$and $g:\left(B_{+}, B_{-}\right) \rightarrow\left(C_{+}, C_{-}\right)$is 


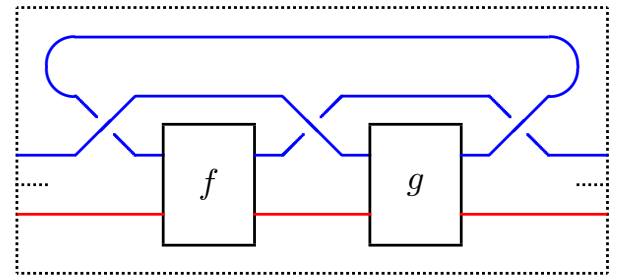

The tensor product of $\left(A_{+}, A_{-}\right)$and $\left(B_{+}, B_{-}\right)$is $\left(A_{+} \otimes B_{+}, B_{-} \otimes A_{-}\right)$, while the unit object is $(I, I)$; see (Joyal et al. 1996; Hasegawa 2009) for further details of the structure of $\operatorname{Int}(\mathcal{C})$.

Proposition 4.2. For a Hopf algebra $A=(A, m, 1, \Delta, \epsilon, S)$ with an invertible antipode $S$ in a traced symmetric monoidal category $\mathcal{C}$, there is a quasi-triangular Hopf algebra $\left((A, A), m^{\mathrm{d}}, 1^{\mathrm{d}}, \Delta^{\mathrm{d}}, \epsilon^{\mathrm{d}}, S^{\mathrm{d}}\right)$ with a universal $R$-matrix $R$ in $\operatorname{Int}(\mathcal{C})$ given as follows.

$m^{\mathrm{d}}:(A, A) \otimes(A, A) \rightarrow(A, A)$

$$
\Delta^{\mathrm{d}}:(A, A) \rightarrow(A, A) \otimes(A, A)
$$

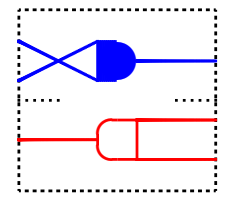

$S^{\mathrm{d}}:(A, A) \rightarrow(A, A)$

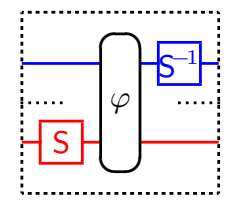

$R: I \rightarrow(A, A) \otimes(A, A)$

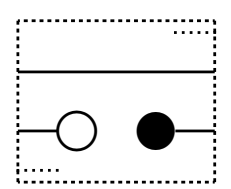

$R^{\circ}: I \rightarrow(A, A) \otimes(A, A)$

$\epsilon^{\mathrm{d}}:(A, A) \rightarrow I$

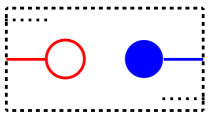

$1^{\mathrm{d}}: I \rightarrow(A, A)$
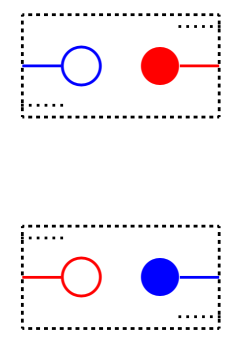

where $\varphi: A \otimes A \rightarrow A \otimes A$ is

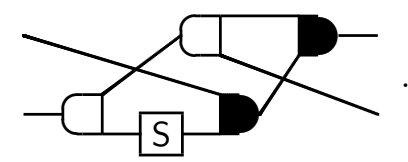

When $\mathcal{C}$ itself is a compact closed category, there is a strong symmetric monoidal equivalence $F: \operatorname{Int}(\mathcal{C}) \rightarrow \mathcal{C}$ sending $\left(A_{+}, A_{-}\right)$to $F\left(A_{+}, A_{-}\right)=A_{-}^{*} \otimes A_{+}$, with the obvious isomorphism from $F\left(A_{+}, A_{-}\right) \otimes F\left(B_{+}, B_{-}\right)=A_{-}^{*} \otimes A_{+} \otimes B_{-}^{*} \otimes B_{+}$to $F\left(\left(A_{+}, A_{-}\right) \otimes\right.$ $\left.\left(B_{+}, B_{-}\right)\right)=\left(B_{-} \otimes A_{-}\right)^{*} \otimes A_{+} \otimes B_{-}$. Via this equivalence, this quasi-triangular Hopf algebra on $(A, A)$ in $\operatorname{Int}(\mathcal{C})$ is sent to a quasi-triangular Hopf algebra on $A \otimes A^{*}$ in $\mathcal{C}$, as claimed in Proposition 4.1. 


\subsection{Quantum double of $\bar{G}$ in $\mathbf{R e l}$}

Now we turn our attention to the Hopf algebra $\bar{G}$ in Rel. Since the antipode $S$ of $\bar{G}$ is invertible, we can apply the quantum double construction to $\bar{G}$, and we obtain a quasi-triangular (in fact, ribbon) Hopf algebra $D(\bar{G})$.

By Proposition 4.2, the quantum double of $\bar{G} \operatorname{in} \operatorname{Int}(\operatorname{Rel})$ is $\left((G, G), m^{\mathrm{d}}, 1^{\mathrm{d}}, \Delta^{\mathrm{d}}, \epsilon^{\mathrm{d}}, S^{\mathrm{d}}, R\right)$ where

$$
\begin{aligned}
m^{\mathrm{d}} & =\left\{\left(\left(\left(h_{1}, h_{2}\right), g\right),\left(h_{1} h_{2},\left(h_{1}^{-1} g h_{1}, g\right)\right)\right) \mid g, h_{1}, h_{2} \in G\right\} \\
1^{\mathrm{d}} & =\{((*, g),(e, *)) \mid g \in G\} \\
\Delta^{\mathrm{d}} & =\left\{\left(\left(h,\left(g_{2}, g_{1}\right)\right),\left((h, h), g_{1} g_{2}\right)\right) \mid g_{1}, g_{2}, h \in G\right\} \\
\epsilon^{\mathrm{d}} & =\{((g, *),(*, e)) \mid g \in G\} \\
S^{\mathrm{d}} & =\left\{\left(\left(h, h^{-1} g^{-1} h\right),\left(h^{-1}, g\right)\right) \mid g, h \in G\right\} \\
R & =\{((*,(h, g)),((e, g), *)) \mid g, h \in G\} \\
R^{\circ} & =\left\{\left(\left(*,\left(h, g^{-1}\right)\right),((e, g), *)\right) \mid g, h \in G\right\}
\end{aligned}
$$
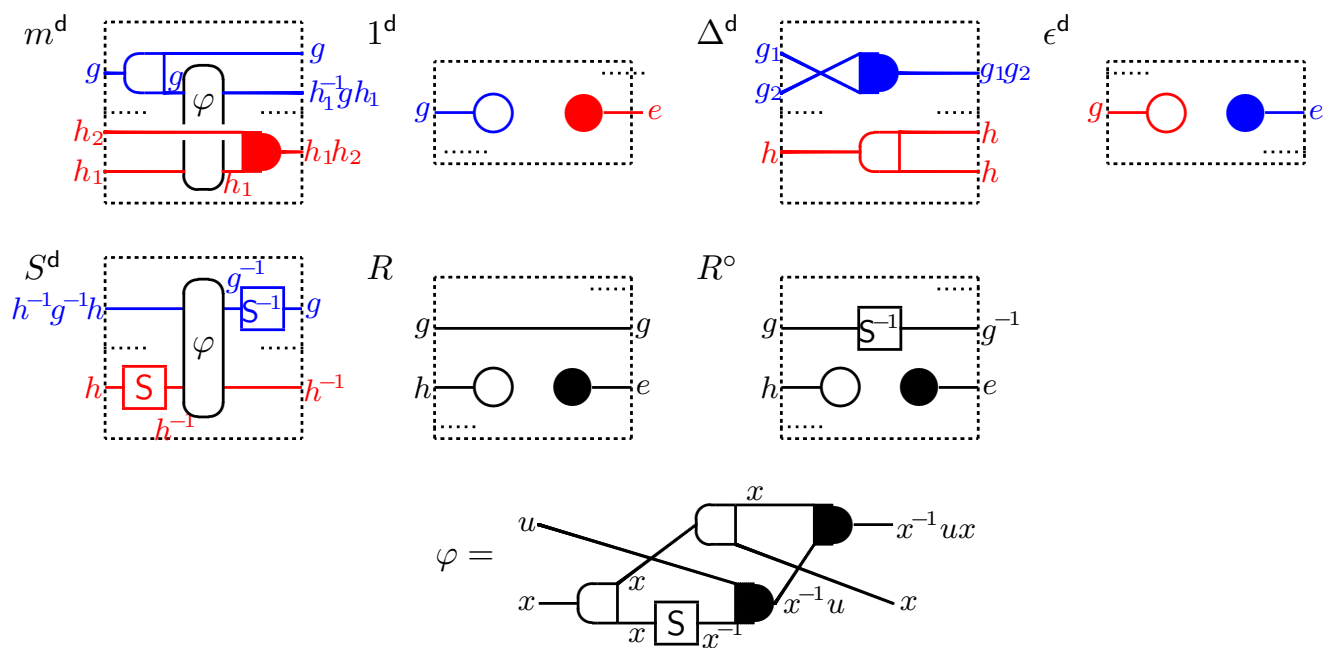

Via the strong symmetric monoidal equivalence from Int( $\operatorname{Rel})$ to $\mathbf{R e l}$, we obtain:

Theorem 4.1. Suppose that $G=\left(G, \cdot, e,(-)^{-1}\right)$ is a group. There is a ribbon Hopf algebra $D(\bar{G})=\left(G \times G, m^{\mathrm{d}}, 1^{\mathrm{d}}, \Delta^{\mathrm{d}}, \epsilon^{\mathrm{d}}, S^{\mathrm{d}}, R, v\right)$ in Rel, with

$$
\begin{aligned}
m^{\mathrm{d}} & =\left\{\left(\left(\left(g, h_{1}\right),\left(h_{1}^{-1} g h_{1}, h_{2}\right)\right),\left(g, h_{1} h_{2}\right)\right) \mid g, h_{1}, h_{2} \in G\right\} \\
1^{\mathrm{d}} & =\{(*,(g, e)) \mid g \in G\} \\
\Delta^{\mathrm{d}} & =\left\{\left(\left(g_{1} g_{2}, h\right),\left(\left(g_{1}, h\right),\left(g_{2}, h\right)\right) \mid g_{1}, g_{2}, h \in G\right\}\right. \\
\epsilon^{\mathrm{d}} & =\{((e, g), *) \mid g \in G\} \\
S^{\mathrm{d}} & =\left\{\left((g, h),\left(h^{-1} g^{-1} h, h^{-1}\right)\right) \mid g, h \in G\right\} \\
R & =\{(*,((g, e),(h, g))) \mid g, h \in G\} \\
v & =\{(*,(g, g)) \mid g \in G\}
\end{aligned}
$$

where $R$ is the universal $R$-matrix and $v$ is the universal twist.

When $G$ is not Abelian, $D(\bar{G})$ is neither commutative nor co-commutative. Below we shall observe that modules of $D(\bar{G})$ can be identified with the crossed $G$-sets (Freyd and Yetter 1989; Whitehead 1949). 


\section{A ribbon category of crossed $G$-sets}

\subsection{Crossed $G$-sets}

Let $G=\left(G, \cdot, e,(-)^{-1}\right)$ be a group. A crossed $G$-set $X=(X, \bullet,|-|)$ is given by a set $X$ together with a group action $\bullet: G \times X \rightarrow X$ and a function |_| from $X$ to $G$ such that, for any $g \in G$ and $x \in X,|g \bullet x|=g \cdot|x| \cdot g^{-1}$ holds. For instance, $G$ itself can be seen a crossed $G$-set with $g \bullet h=g \cdot h \cdot g^{-1}$ and $|h|=h$. Another trivial example is a $G$-set with $|x|=e$.

Proposition 5.1. For any set $X$, there is a bijective correspondence between $D(\bar{G})$ modules on $X$ and crossed $G$-sets on $X$.

Indeed, if $\alpha: G \times G \times X \rightarrow X$ is a $D(\bar{G})$-module, for any $g \in G$ and $x \in X$ there are unique $h \in G$ and $y \in X$ such that $(((h, g), x), y) \in \alpha$, and $X$ carries the structure of crossed $G$-set where $g \bullet x$ is this uniquely determined $y$ and $|x|$ is the unique $h$ such that $(((h, e), x), x) \in \alpha$. Conversely, a crossed $G$-set $(X, \bullet,|-|)$ gives rise to a module $\{(((|g \bullet x|, g), x), g \bullet x) \mid g \in G, x \in X\}: G \times G \times X \rightarrow X$. It is not hard to see that this is a bijective correspondence.

A morphism of crossed $G$-sets from $\left(X, \bullet,||_{-}\right)$to $\left(Y, \bullet,||_{-}\right)$, corresponding to the morphism of $D(\bar{G})$-modules, is a binary relation $r: X \rightarrow Y$ such that $(x, y) \in r$ implies $(g \bullet x, g \bullet y) \in r$ as well as $|x|=|y|$. The identity and composition of morphisms are just the same as those of binary relations. Let us denote the category of crossed $G$-sets and morphisms by $\operatorname{XRel}(G)$ which is isomorphic to $\operatorname{Mod}(D(\bar{G}))$. We note that the category $G$-XXSf of crossed $G$-sets of Freyd and Yetter (Freyd and Yetter 1989) is the subcategory of $\operatorname{XRel}(G)$ whose morphisms are restricted to functions and objects are restricted to finite ones. A variant of $\operatorname{XRel}(G)$ where $G$ is not a group but a commutative monoid has appeared in (Abramsky et al. 1999).

For any set $X$, the free crossed $G$-set over $X$ is given by $\mathcal{F}(X)=\left(G \times G \times X, \bullet,||_{-}\right)$ with $g \bullet\left(h_{1}, h_{2}, x\right)=\left(g \cdot h_{1} \cdot g^{-1}, g \cdot h_{2}, x\right)$ and $\left|\left(h_{1}, h_{2}, x\right)\right|=h_{1} . \mathcal{F}$ extends to a functor from $\operatorname{Rel}$ to $\operatorname{XRel}(G)$ which is left adjoint to the forgetful functor $\mathcal{U}: \operatorname{XRel}(G) \rightarrow \operatorname{Rel}$ which sends $(X, \bullet,|-|)$ to $X$.

\subsection{The ribbon structure on $\operatorname{XRel}(G)$}

By Proposition 2.1, $\operatorname{Mod}(D(\bar{G}))$, hence $\operatorname{XRel}(G)$, is a ribbon category. In $\mathbf{X R e l}(G)$, the tensor unit is $I=(\{*\},(g, x) \mapsto x, x \mapsto e)$, and the tensor product of $X=(X, \bullet,|-|)$ and $Y=(Y, \bullet,|-|)$ is

$$
X \otimes Y=(X \times Y,(g,(x, y)) \mapsto(g \bullet x, g \bullet y),(x, y) \mapsto|x| \cdot|y|) .
$$

The tensor product of morphisms, as well as the coherence isomorphisms $a, l, r$, are inherited from Rel. For this monoidal structure we have a braiding $\sigma_{X, Y}: X \otimes Y \stackrel{\simeq}{\rightarrow} Y \otimes X$ induced by the universal $R$-matrix $R$ as

$$
\sigma_{X, Y}=\{((x, y),(|x| \bullet y, x)) \mid x \in X, y \in Y\} .
$$


There is a twist $\theta_{X}: X \stackrel{\simeq}{\rightarrow} X$ induced by the universal twist $v$ :

$$
\theta_{X}=\{(x,|x| \bullet x) \mid x \in X\} .
$$

For a crossed $G$-set $X=(X, \bullet,|-|)$, its left dual is $X^{*}=\left(X, \bullet,||^{-1}\right)$, with unit $\eta_{X}=$ $\{(*,(x, x)) \mid x \in X\}: I \rightarrow X \otimes X^{*}$ and counit $\varepsilon_{X}=\{((x, x), *) \mid x \in X\}: X^{*} \otimes X \rightarrow I$. We note that the canonical trace on $\operatorname{XRel}(G)$ is given just like that on $\operatorname{Rel}$ : for $f$ : $A \otimes X \rightarrow B \otimes X$, its trace $\operatorname{Tr}_{A, B}^{X} f: A \rightarrow B$ is

$$
\operatorname{Tr}_{A, B}^{X} f=\{(a, b) \in A \times B \mid \exists x \in X((a, x),(b, x)) \in f\} .
$$

\subsection{Interpreting tangles in $\operatorname{XRel}(G)$}

Since the category of (oriented, framed) tangles is equivalent to the ribbon category freely generated by a single object (Shum 1994), by specifying a ribbon category and an object, we always obtain a structure-preserving functor from the category of tangles to the ribbon category, which determines an invariant of tangles (Yetter 2001). This is also the case for $\operatorname{XRel}(G)$.

For understanding how a crossed $G$-set gives rise to an invariant of tangles, it is helpful to consider the rack (Fenn and Rourke 1992) associated to the crossed $G$-set ${ }^{\ddagger}$. Given a crossed $G$-set $(X, \bullet,|| \mid)$, let us define operators $\triangleright, \triangleright^{-1}: X \times X \rightarrow X$ as $x \triangleright y=|y| \bullet x$ and $x \triangleright^{-1} y=|y|^{-1} \bullet x$. Then $\left(X, \triangleright, \triangleright^{-1}\right)$ forms a rack; that is, the following equations hold ${ }^{\S}$.

$$
\begin{array}{cl}
(x \triangleright y) \triangleright^{-1} y=x=\left(x \triangleright^{-1} y\right) \triangleright y & \text { (bijectivity of }(-) \triangleright y) \\
(x \triangleright y) \triangleright z=(x \triangleright z) \triangleright(y \triangleright z) & \text { (self-distributivity) }
\end{array}
$$

Now the braiding and twist can be described in terms of this rack: $\sigma_{X, Y}=\{((x, y),(y \triangleright$ $x, x)) \mid x \in X, y \in Y\}$ and $\theta_{X}=\{(x, x \triangleright x) \mid x \in X\}$. The interpretation of a tangle diagram in $\operatorname{XRel}(G)$ with a crossed $G$-set $X$ is then determined by all possible $X$ labellings of the segments from an underpass to the next underpass satisfying " $y$ under $x$ from left gives $y \triangleright x$ " and " $y$ under $x$ from right gives $y \triangleright^{-1} x$ ".
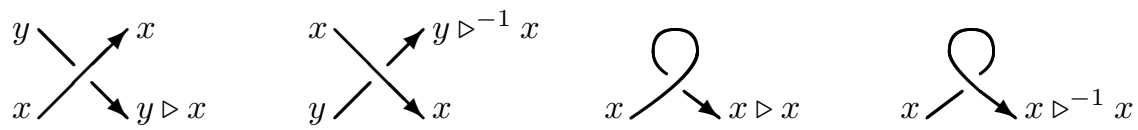

For instance, the self-distributivity justifies the Reidemeister move III:
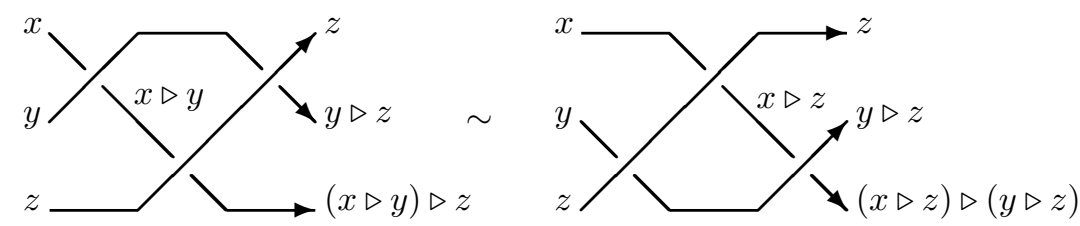

Similarly, the Reidemeister move II is justified by the bijectivity:

\footnotetext{
$\ddagger$ Indeed, another name for crossed $G$-sets coined by Fenn and Rourke is augmented racks. They have shown that every rack arises from an augmented rack, hence a crossed $G$-set.

$\S$ However, this does not have to be a quandle in the sense of Joyce (Joyce 1982), since the idempotency $x \triangleright x=x$ does not hold in general.
} 


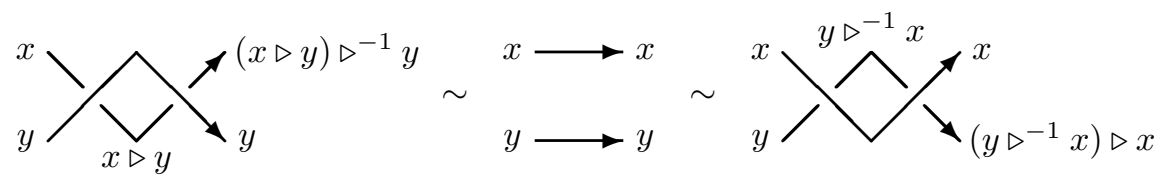

The framed version of Reidemeister move I is also justified by the self-distributivity and bijectivity:

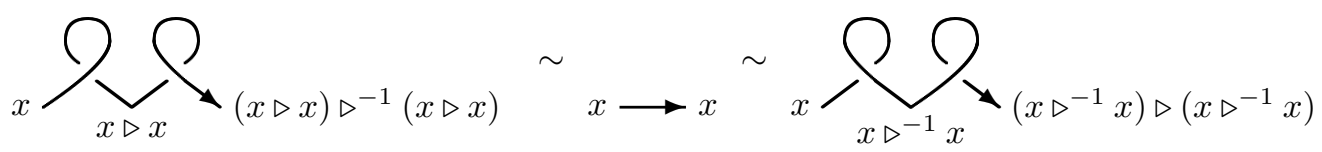

Example 5.1. Consider of the following link:

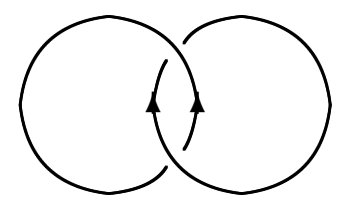

Its interpretation in $\operatorname{XRel}(G)$ with a crossed $G$-set $X$ takes a value in $\operatorname{XRel}(G)(I, I)=$ $\left\{i d_{I}, \emptyset\right\}$, and it is the identity relation $i d_{I}$ if there exist $x, y \in X$ such that $x=x \triangleright y$ and $y=y \triangleright x$ hold; otherwise it is the empty relation $\emptyset$.

These invariants are far from complete. For example, the links $\bigcirc \bigcirc$ and $\bigcirc$ always have the same interpretation for any crossed $G$-set.

\section{A model of braided linear logic}

In this section, we outline the notion of models of (fragments of) braided linear logic, and see how $\operatorname{XRel}(G)$ in the previous section gives such a model. For a detailed exposition on categorical models of linear logic, see (Melliès 2009).

\subsection{Models of braided linear logic}

By a model of braided multiplicative linear logic (braided MLL), we mean a braided *autonomous category (Barr 1995); note that a ribbon category is braided $*$-autonomous, hence is a model of braided MLL. A model of braided multiplicative additive linear logic (braided MALL) is then a braided $*$-autonomous category with finite products.

For exponential, we employ the following generalization of the notion of linear exponential comonads (Hyland and Schalk 2003) on symmetric monoidal categories: by a linear exponential comonad on a braided monoidal category we mean a braided monoidal comonad whose category of coalgebras is a category of commutative comonoids. A model of braided MELL is then a braided $*$-autonomous category with a linear exponential comonad. (An implication of this definition is that braiding becomes symmetry on exponential objects: $\left.\sigma_{! X, ! Y}^{-1}=\sigma_{! Y, ! X}.\right)$ A model of braided LL is a model of MALL with a linear exponential comonad (or a model of MELL with finite products). 


\section{2. $\mathbf{X R e l}(G)$ as a model of braided linear logic}

$\operatorname{XRel}(G)$ is a ribbon category with finite products, hence is a model of braided MALL.

There is a strict balanced monoidal functor $F: \operatorname{Rel} \rightarrow \operatorname{XRel}(G)$ which sends a set $X$ to $F X=(X,(g, x) \mapsto x, x \mapsto e) . F$ has a right adjoint $U: \mathbf{X R e l}(G) \rightarrow \operatorname{Rel}$ which sends $X=\left(X, \bullet,||_{-}\right)$to $U X=\{x \in X|| x \mid=e\} / \sim$ where $x \sim y$ iff $g \bullet x=y$ for some $g$. By composing $F$ and $U$ with a linear exponential comonad ! on Rel (e.g. the finite multiset comonad), we obtain a linear exponential comonad $F ! U$ on $\operatorname{XRel}(G)$ whose category of coalgebras is equivalent to that of !. Hence $\operatorname{XRel}(G)$ is a model of braided LL. As a result, there exists a linear fixed-point operator on $\operatorname{XRel}(G)$ as given in (Hasegawa 2009).

$\operatorname{XRel}(G)$ is degenerate as a model of LL in the sense that it cannot distinguish tensor from par. As an easy remedy, one may apply the simple self-dualization construction (Hyland and Schalk 2003) for obtaining a "non-compact" model. For a braided monoidal closed category $\mathcal{C}$ with finite products, there is a braided $*$-autonomous structure on $\mathcal{C} \times \mathcal{C}^{\text {op }}$ whose tensor unit is $(I, 1)$ (where 1 is a terminal object and should not be confused with the unit element of a monoid) and tensor product is given by $(U, X) \otimes(V, Y)=$ $(U \otimes V, U \multimap Y \times V \multimap X)$, while the duality is given by $(U, X)^{\perp}=(X, U)$. By applying the simple self-dualization construction to $\operatorname{XRel}(G)$ we obtain a "non-compact" model $\mathbf{X R e l}(G) \times \mathbf{X} \operatorname{Rel}(G)$ op of braided LL. Alternatively, $\mathbf{X} \operatorname{Rel}(G) \times \operatorname{XRel}(G)^{\text {op }}$ arises as the category of modules of $D(\bar{G})$ (or $(D(\bar{G}), \emptyset)$ to be more precise) in the $*$-autonomous category $\mathbf{R e l} \times \mathbf{R e l}^{\mathrm{op}}$ obtained by the simple self-dualization on Rel.

\section{Concluding remarks}

We have demonstrated that there are many non-trivial Hopf algebras in the category of sets and binary relations. In particular, by applying the quantum double construction we have constructed a non-commutative non-co-commutative Hopf algebra with a universal $R$-matrix and a universal twist, and the ribbon category of its modules turns out to be a category of crossed $G$-sets.

Technically, most of our results are variations or instances of the already established theory of quantum groups, and we do not claim much novelty in this regard. What is much more important in this work, we believe, is that our results show that it is indeed possible to carry out a substantial part of quantum group theory in a category used for semantics of computation and logic. Although we have spelled out just a particular case of Rel, we expect that the same can be done meaningfully in various other settings, including

- the *-autonomous category of coherent spaces and linear stable maps (Girard 1987), and its variations used as models of linear logic,

- various categories of games, in particular the compact closed category of Conway games (Joyal 1977; Melliès 2004), and

- the category of sets (or presheaves on discrete categories) and linear normal functors (Hasegawa 2002), as well as the bicategory of small categories and profunctors.

The first two would lead to models of braided linear logic and some braided variants of 
game semantics. The third should be a direct refinement of our work on Rel, in that we replace binary relations $X \times Y \rightarrow 2$ with Set-valued functors $X \times Y \rightarrow$ Set (which amount to linear normal functors from $\mathbf{S e t}^{X}$ to $\operatorname{Set}^{Y}$ ).

Finally, we must admit that the computational significance of braided monoidal structure is yet to be examined. As far as we know, $\operatorname{XRel}(G)$ is the first non-symmetric ribbon category featuring a linear exponential comonad, allowing non-trivial interpretations of braidings as well as recursive programs at the same time. If we are to develop a sort of braided variant of denotational semantics in future, $\mathbf{X R e l}(G) \operatorname{might}$ be a good starting point. A potentially related direction would be the area of topological quantum computation (Freedman et al. 2002; Kitaev 2003; Wang 2010; Panangaden and Paquette 2011), in which modular tensor categories (semisimple ribbon categories with finite simple objects satisfying an extra condition) (Turaev 1994; Bakalov and Kirilov 2001) play the central role. Although $\mathbf{X R e l}(G)$ is not modular, it might be possible to develop a toy (and suitably simplified) model of topological quantum computation in it.

\section{Acknowledgements}

I thank Shin-ya Katsumata and Craig Pastro for helpful discussions. This work was partly supported by the Grant-in-Aid for Scientific Research (C) 20500010.

\section{References}

Abramsky, S., Blute, R. and Panangaden, P. (1999) Nuclear and trace ideals in tensored *categories. J. Pure Appl. Algebra 143, 3-47.

Abramsky, S. and Coecke, B. (2004) A categorical semantics of quantum protocols, In Proc. 19th IEEE Symposium on Logic in Computer Science, pp.415-425.

Abramsky, S., Haghverdi, E. and Scott, P.J. (2002) Geometry of Interaction and linear combinatory algebras. Math. Struct. Comput. Sci. 12, 625-665.

Bakalov, B. and Kirilov, A. (2001) Lectures on Tensor Categories and Modular Functors. University Lecture Series 21, American Mathematical Society.

Barr, M. (1995) Nonsymmetric *-autonomous categories. Theoret. Comput. Sci. 139, 115-130.

Bloom, S. and Ésik, Z. (1993) Iteration Theories. EATCS Monographs on Theoretical Computer Science, Springer-Verlag.

Blute, R. (1996) Hopf algebras and linear logic. Math. Struct. Comput. Sci. 6, 189-217.

Bruguieres, A. and Virelizier, A. (2007) Hopf monads. Adv. Math. 215, 679-733.

Chen, H.-X. (2000) Quantum doubles in monoidal categories. Comm. Algebra 28, 2303-2328.

Cockett, J.R.B. and Seely, R.A.G. (1997) Weakly distributive categories. J. Pure Appl. Algebra 114, 133-173.

Drinfel'd, V.G. (1987) Quantum groups. In Proc. Intrn. Congr. Math., Berkley, 1986, vol. 1, pp. $798-820$.

Fenn, R. and Rourke, C. (1992) Racks and links in codimension two. J. Knot Theory Ramifications 1, 343-406.

Freedman, M.H., Kitaev, A. and Wang, Z. (2002) Simulation of topological field theories by quantum computers. Commun. Math. Phys. 227, 587-603.

Freyd, P. and Yetter, D.N. (1989) Braided compact closed categories with applications to low dimensional topology. Adv. Math. 77, 156-182. 
Girard, J.-Y. (1987) Linear logic. Theoret. Comput. Sci. 50, 1-102.

Girard, J.-Y. (1989) Geometry of Interaction I: interpretation of system F. in Proc. Logic Colloquium '88, pp. 221-260.

Haghverdi, E. and Scott, P.J. (2011) Geometry of Interaction and the dynamics of proof reduction: a tutorial. In New Structures for Physics, Springer Lecture Notes in Phys. 813, pp. $357-417$.

Hasegawa, M. (1999) Models of Sharing Graphs: A Categorical Semantics of let and letrec. Distinguished Dissertations Series, Springer-Verlag.

Hasegawa, M. (2009) On traced monoidal closed categories. Math. Struct. Comput. Sci. 19, $217-244$.

Hasegawa, M. (2010) Bialgebras in Rel. In Proc. Mathematical Foundations of Programming Semantics, Electr. Notes Theor. Comput. Sci. 265, pp. 337-359.

Hasegawa, R. (2002) Two applications of analytic functors. Theoret. Comput. Sci. 272, 113-175.

Hildebrandt, T.T., Panangaden, P. and Winskel, G. (2004) A relational model of nondeterministic dataflow. Math. Struct. Comput. Sci. 14, 613-649.

Hyland, J.M.E. and Schalk, A. (2003) Glueing and orthogonality for models of linear logic. Theoret. Comp. Sci. 294, 183-231.

Joyal, A. (1977) Remarques sur la théorie des jeux à deux personnes. Gazette des Sciences Mathématiques du Québec 1, 46-52.

Joyal, A. and Street, R. (1991) The geometry of tensor calculus, I. Adv. Math. 88, 55-113.

Joyal, A. and Street, R. (1993) Braided tensor categories. Adv. Math. 102, 20-78.

Joyal, A., Street, R. and Verity, D. (1996) Traced monoidal categories. Math. Proc. Cambridge Phils. Soc. 119 (1996), 447-468.

Joyce, D. (1982) A classifying invariant of knots, the knot quandle. J. Pure Appl. Algebra 23, $37-65$.

Kassel, C. (1995) Quantum Groups. Graduate Texts in Mathematics 155, Springer-Verlag.

Kassel, C. and Turaev, V.G. (1995) Double construction for monoidal categories. Acta Math. 175, 1-48.

Katsumata, S. (2008) Attribute grammars and categorical semantics, In Proc. International Colloquium on Automata, Languages and Programming, Springer Lecture Notes in Comput. Sci. 5126, pp. 271-282.

Kelly, G.M. and Laplaza, M.L. (1980) Coherence for compact closed categories. J. Pure Appl. Algebra 19, 193-213.

Kitaev, A. (2003) Fault-tolerant quantum computation by anyons. Ann. Phys. 303, 3-20.

Mac Lane, S. (1971) Categories for the Working Mathematician. Graduate Texts in Mathematics 5, Springer-Verlag.

Majid, S. (1990) Physics for algebraists: noncommutative and noncocommutative Hopf algebras by a bicrossproduct construction. J. Algebra 130, 17-64.

Majid, S. (1994) Algebras and Hopf algebras in braided categories. In Advances in Hopf Algebras, Lec. Notes Pure and Applied Maths. 158, 55-105.

Majid, S. (1995) Foundations of Quantum Group Theory. Cambridge University Press.

Melliès, P.-A. (2004) Asynchronous games 3: an innocent model of linear logic. In Proc. Category Theory and Computer Science, Electr. Notes Theor. Comput. Sci. 122, pp. 171-192.

Melliès, P.-A. (2009) Categorical semantics of linear logic. In Interactive Models of Computation and Program Behaviour, Panoramas et Synthèses 27, pp. 1-196, Société Mathématique de France.

Panangaden, P. and Paquette, É.O. (2011) A categorical presentation of quantum computation with anyons. In New Structures for Physics, Springer Lecture Notes in Phys. 813, pp. 9831025. 
Pastro, C. and Street, R. (2009) Closed categories, star-autonomy, and monoidal comonads. J. Algebra 321, 3494-3520.

Selinger, P. (2011) A survey of graphical languages for monoidal categories. In New Structures for Physics, Springer Lecture Notes in Phys. 813, pp. 289-355.

Shum, M.-C. (1994) Tortile tensor categories. J. Pure Appl. Algebra 93, 57-110.

Ştefănescu, G. (2000) Network Algebra. Series in Discrete Mathematics and Theoretical Computer Science, Springer-Verlag.

Street, R. (2007) Quantum Groups: A Path to Current Algebra. Australian Mathematical Society Lecture Series 19, Cambridge University Press.

Takeuchi, M. (1971) Free Hopf algebras generated by coalgebras. J. Math. Soc. Japan 23, 561582.

Takeuchi, M. (1999) Finite Hopf algebras in braided tensor categories. J. Pure Appl. Algebra 138, 59-82.

Turaev, V.G. (1994) Quantum Invariants of Knots and 3-Manifolds. de Gruyter.

Wang, Z. (2010) Topological Quantum Computation. CBMS Regional Conference Series in Mathematics 112, American Mathematical Society.

Whitehead, J.H.C. (1949) Combinatorial homotopy. II. Bull. Amer. Math. Soc. 55, 453-496.

Yetter, D.N. (2001) Functorial Knot Theory. World Scientific. 